\title{
STABILIZATION VERSUS MCKENZIE EXERCISES IN PATIENTS WITH MECHANICAL NECK DYSFUNCTION
}

\section{Tarek Ammar}

Assistant Professor, Department of Basic Science, Faculty of Physical Therapy, Cairo University.

\section{ABSTRACT}

Background: Mechanical neck dysfunction (MND) is a disabling and costly disorder. There are insufficient data available to determine the efficacy of the stabilization and McKenzie exercises on MND.

Purpose: The objective of this study was to compare stabilization exercises to McKenzie exercises in patients with MND.

Design: Two group pretest-posttest design.

Materials and Methods: Twenty-seven participants were randomly allocated to the stabilization group ( $n=14)$ and the McKenzie group ( $n=13$ ). Each group received treatment twice a week over a 6-week period. Outcome included pain intensity measured on a visual analogue scale, disability measured with the neck disability index, pain threshold measured with the pressure algometer and cervical range of motion measured with an electronic goniometer at baseline and after six weeks of treatment.

Results: Pain intensity, functional disability, pain threshold and cervical range of motion showed significant differences in all groups after intervention. However, there were no significant differences between groups.

Conclusion: Within the limitations of this study, both programs were equally effective in improving pain, disability, and range of motion of patients with MND.

KEY WORDS: Neck dysfunction, Exercises, Stabilization, McKenzie.

Address for correspondence: Tarek Ammar, PhD, PT, Assistant Professor, Faculty of Physical Therapy, Cairo University, 7 Ahmed Elzyat St. Ben Elsaryat, El Dokki, Giza, Egypt 12612

Phone: 011202-37617691 E-Mail: tarekphysicaltherapy@cu.edu.eg

Access this Article online

Quick Response code

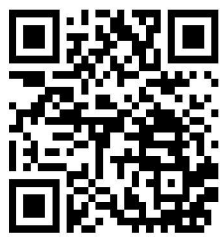

DOI: $10.16965 /$ ijpr.2017.256

\begin{tabular}{|c|c|c|}
\hline \multicolumn{3}{|c|}{ Journal Information } \\
\hline \multicolumn{3}{|c|}{$\begin{array}{l}\text { International Journal of Physiotherapy and Research } \\
\text { V for 2016 ISSN (E) 2321-1822 | ISSN (P) 2321-8975 }\end{array}$} \\
\hline \multicolumn{3}{|c|}{ Article Information } \\
\hline $\begin{array}{l}\text { Received: } 29 \text { Oct } 2017 \\
\text { Peer Review: } 01 \text { Nov } 2017 \\
\text { Revised: None }\end{array}$ & $\begin{array}{l}\text { Accepted: } 08 \text { Dec } \\
\text { Published (O): } 11 \\
\text { Published (P): } 11\end{array}$ & $\begin{array}{l}7 \\
2018 \\
2018\end{array}$ \\
\hline
\end{tabular}

complain of intermittent pain, restriction of end range of movement and muscle dysfunction [5]. Physical Therapists use various approaches to treat MND. They include but not limited to electrical therapy, laser, manual therapy, manipulation, hot packs, home exercise programs, advice on posture, muscle stretching techniques, deep neck flexor stabilizing exercise and other modalities [6-8]. Despite the large amount of evidence regarding management of MND, there is insufficient evidence in the optimal treatment approach. It is highly recommended to develop 
a universal classification system for patients with MND.

Stabilization and endurance exercises have been recommended as an effective intervention for patients with neck pain [9]. McKenzie exercises have also been also recommended to reduce spinal pain [10]. Stabilization and McKenzie exercises utilize different protocols to reduce pain and there is controversy about which is more effective in spinal pain [11]. Stabilization exercise works to rectify muscle abnormalities and restore correct function of muscles to support and stabilize the spine $[10,11]$. Whereas the McKenzie method involves postural awareness and repetitive movements with the fundamental concept of a reverse force may decrease pain and restore function [12].

A few studies on the impact of stabilization or McKenzie exercises in MND may have been done. However, there are no randomized controlled trials on a correlation or comparison between the two stabilization and McKenzie programs in any sample of patients with MND. Therefore, this study was designed to compare the pain perception, disability, pain threshold, and range of motion (ROM) between stabilization and McKenzie exercises applied to patients with MND.

\section{MATERIALS AND METHODS}

Design: This research has a pretest-posttest control group design. Participants were randomly allocated to one of two groups: (1) a group that received the conservative physical therapy program and stabilization exercises. (2) a group that received the conservative physical therapy program and McKenzie exercises. The research physical therapist used a table of random numbers to assign participants to both groups. He performed the outcome measurements of participants at the baseline and final sessions. He was unaware of group allocation. However, the clinical therapist who administered the exercises was aware of group allocation. Participants in both groups received two sessions per week for six weeks.

Participants: Fifty-two participants with MND were recruited from a physical therapy outpatient setting. Participants of any race or gender with a chief complaint of a neck disability index of 15 points or more, and neck pain duration of at least 3 months were eligible for the study. Exclusion criteria included a history of orthopedic surgery to the neck and shoulder, neurological symptoms due to cervical dysfunction, trauma, temporomandibular surgery, injection, carcinoma, cardiovascular and neurological conditions. All subjects signed a consent form permitting the use of their data. The treating therapist instructed the participants to refrain from other forms of any other pain modality or medicine during the study. Figure 1 shows the CONSORT flow chart of the participants through this trial.

Instruments

Visual Analogue Scale (VAS): The tester used the VAS to measure current pain intensity, average pain intensity over the past 2-3 days, and the worst level of pain experienced during the past 2-3 days. The tester calculated the mean of the scores to provide a valid and reliable pain measure score [13].

Neck Disability Index (NDI): The tester used the NDI to measure functional disability. It has good internal consistency, reliability, validity and an excellent ability to distinguish patients with different levels of disability [14].

Pressure Algometer: The tester used an algometer (J-tech Medical, Midvale, UT, USA) to measure the pressure pain threshold. The tester applied the algometer at a rate of $1 \mathrm{~kg} / \mathrm{sec}$ vertically to the testing site to measure the pain threshold. The investigator asked each participant to sound 'Ouch' to indicate pain. The tester calculated the mean of the three consecutive measurements on the most severe painful point on the upper trapezius before and after each treatment [15].

Myrin goniometer: The investigator used the Myrin goniometer to assess active neck motions. It is a reliable instrument used to evaluate active neck motions at various times by multiple testers. It may objectively be used to monitor the success of a therapeutic regimen [16].

Pressure biofeedback device (treatment instrument): It was used to quantify and retrain the stabilizing muscles. The device was the sstabilizer pressure biofeedback, Chattanooga group, Chattanooga, USA [17]. 
Procedures: Participants in both groups received the conservative physical therapy program (heat intervention, ultrasound and neck ROM exercises). The first group received the cervical stabilization which included the craniocervical flexion exercises. The exercises aimed to strengthen the upper neck deep flexor muscles. The superficial sternocleidomastoid and anterior scalene muscles were kept relaxed while doing the stabilization [18]. The therapist put the biofeedback device and then flattened cervical lordosis was confirmed by using the visual feedback obtained via the dials of the device. First, the air bag under the neck was inflated to $20 \mathrm{~mm} \mathrm{Hg}$. Then, the participant pressed the bag slightly with slight increments of pressure through the sensor dial (i.e., $2 \mathrm{~mm}$ $\mathrm{Hg}$; up to $30 \mathrm{mmHg}$ ). The subject maintained an isometric hold of up to 10 seconds, and up to 10 repetitions. The therapist asked the participants to repeat each exercise for 10 times with 3 rest periods [17].

The second group received the McKenzie exercises in addition to the conservative program. The McKenzie exercises included head retraction and neck extension in sitting position, head retraction and neck extension in supine position, left and right lateral bending, head turning, and neck flexion in sitting position. Each participant maintained the maximal muscle contraction in each exercise for 7 seconds. Each patient repeated the exercise for 10 to 20 times [19]. Participants of both groups were instructed to perform the home program exercises in the days in which they do not come to the clinic. The investigator used self-report logs to measure adherence of patients to the home exercise programs.

Data Analysis: It was performed using SPSS for Windows, version 18.0. The independent samples t-test was used for comparing the McKenzie and stabilization groups. The paired-t test was used to compare the dependent parameters before and after intervention in each group. Statistical significance for all tests was accepted below the 0.05 level.

\section{RESULTS}

Twelve participants refused to enroll in the study, and 8 patients did not meet the inclusion criteria. Thirty-two participants enrolled into the study. Two patients dropped out in the first group due to time constrains and changing medicine during the study. Three patients dropped out in the second group due to lack of motivation and transportation problems. Patient flow through the study is shown in the CONSORT flow chart shown in the Figure 1.

Fig. 1: Flow chart detailing the study.

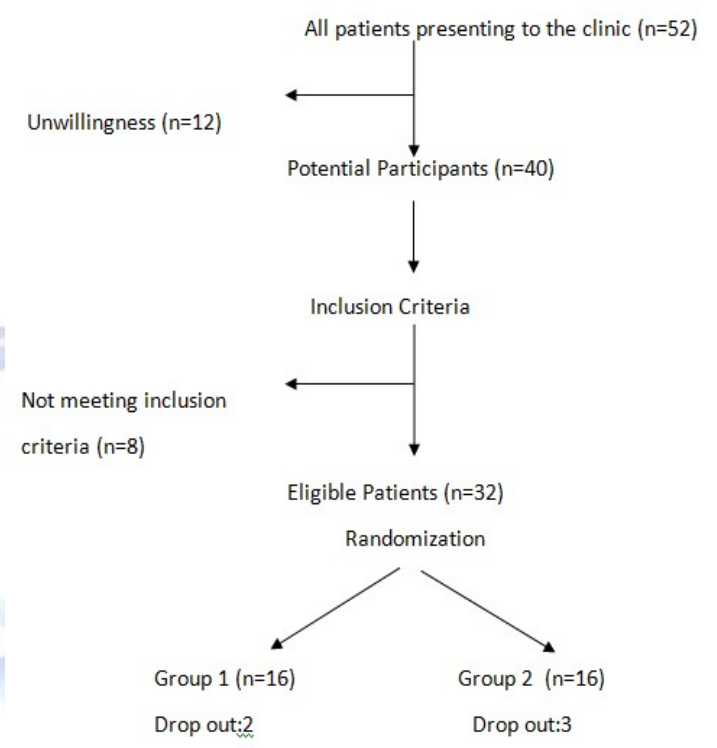

Table 1: Descriptive statistics and t-test for comparing the mean age, weight, height, VAS and NDI of both groups.

\begin{tabular}{|c|c|c|c|c|}
\hline Variables & $\begin{array}{c}\text { Stabilization } \\
\text { group ( } \mathbf{n}=14)\end{array}$ & $\begin{array}{c}\text { McKenzie Ex } \\
\text { group (n=13) }\end{array}$ & $\mathbf{t}$ & $\mathbf{p}$ \\
\hline $\begin{array}{c}\text { Gender } \\
\text { (male/female) }\end{array}$ & $5 / 8$ & $9 / 3$ & & \\
\hline Age (years) & $36.5 \pm 2.9$ & $35.2 \pm 9.8$ & 1.015 & 0.325 \\
\hline Height (cm) & $67.5 \pm 7.0$ & $171.1 \pm 5.0$ & 0.604 & 0.557 \\
\hline Weight (kg) & $69.5 \pm 14.7$ & $74.1 \pm 11.6$ & 0.055 & 0.957 \\
\hline VAS & $6.3 \pm 1.7$ & $5.1 \pm 1.5$ & 1.23 & 0.621 \\
\hline NDI & $14.2 \pm 6.1$ & $13.5 \pm 3.2$ & 0.102 & 0.12 \\
\hline
\end{tabular}

Values are presented as mean (standard deviation). $\mathrm{t}$-test ( $\mathrm{t}), \mathrm{P}$ (probability value) indicates significant value $(P>0.05)$

Table 1 shows the general characteristics of the participants. The author did not find significant differences in the general characteristics between both groups. Differences in pre- and post-test values within groups and between groups are summarized in Tables 2-3.

The stabilization group had significant improvements in VAS, NDI, algometry, cervical extension and lateral neck flexion $(p<0.05)$. Further, the changes in flexion and rotation were not significant. Whereas, the McKenzie group showed 
significant changes in VAS, NDI, algometry, cervical flexion, extension, and lateral neck flexion $(p<0.05)$. However, there were no significant changes in rotation and right lateral flexion. Both groups helped reduce pain perception, disability, pain threshold, increase the ROM, but there were no significant differences between the two groups.

Table 2: Pre and post treatment mean values of VAS, NDI and algometric measurements of both groups (stabilization and McKenzie groups).

\begin{tabular}{|c|c|c|c|c|}
\hline Neck motion & & $\begin{array}{c}\text { Stabilization group } \\
(\mathrm{n}=14)\end{array}$ & $\begin{array}{c}\text { McKenzie Ex group } \\
(\mathrm{n}=13)\end{array}$ & $t \& p$ \\
\hline \multirow{4}{*}{ VAS } & Before & $4.30 \pm 1.60$ & $4.43 \pm 1.44$ & \multirow{4}{*}{$1.409 \& 0.340$} \\
\hline & After & $1.41 \& 0.72$ & $1.81 \& .76$ & \\
\hline & Before-after & $2.20 \& 1.60$ & $1.36 \& 0.52$ & \\
\hline & $t \& p$ & $4.263 \& 0.003^{*}$ & $4.345 \& 0.004^{*}$ & \\
\hline \multirow{4}{*}{ NDI } & Before & $7.15 \pm 1.34$ & $7.20 \& 1.54$ & \multirow{4}{*}{$-0.6790 .351$} \\
\hline & After & $4.01 \pm 1.23$ & $2.45 \& 2.35$ & \\
\hline & Before-after & $3.72 \& 2.57$ & $2.30 \& 2.42$ & \\
\hline & $t \& p$ & $3.52 \pm 0.003^{*}$ & $2.760 \pm 0.042^{*}$ & \\
\hline \multirow{4}{*}{ Algometer Rt } & Before & $9.61 \& 1.26$ & $7.46 .5 \& 4.31$ & \multirow{4}{*}{$1.024 \& .0 .438$} \\
\hline & After & $13.01 \& 2.80$ & $14.1 \& 3.61$ & \\
\hline & Before-after & $1.89 \pm 0.29$ & $2.72 \pm .1 .47$ & \\
\hline & $t \& p$ & $3.478 \& .005^{*}$ & $3.4211 \& 0.006^{*}$ & \\
\hline \multirow{4}{*}{ Algometer Lt } & Before & $8.32 \pm 2.13$ & $7.93 \pm 2.82$ & \multirow{4}{*}{$0.31 \& 0.725$} \\
\hline & After & $13.64 \pm 3.15$ & $13.20 \pm 4.38$ & \\
\hline & Before-after & $2.62 \pm 0.62$ & $2.37 \& 2.36$ & \\
\hline & $t \& p$ & $3.127 \& 0.001^{*}$ & $2.412 \& 0.003$ & \\
\hline
\end{tabular}

Values are presented as mean (standard deviation). Lt: left, Rt: right, t-test $(\mathrm{t}), \mathrm{P}$ (probability value) * indicates significant value $(P<0.05)$

Table 3: Pre and post treatment mean values of ROM of neck both groups (stabilization and McKenzie groups).

\begin{tabular}{|c|c|c|c|c|}
\hline Neck motion & & $\begin{array}{c}\text { Stabilization group } \\
(\mathrm{n}=14)\end{array}$ & $\begin{array}{c}\begin{array}{c}\text { McKenzie Ex group } \\
(n=13)\end{array} \\
\end{array}$ & $t \& p$ \\
\hline \multirow{4}{*}{ VAS } & Before & $4.30 \pm 1.60$ & $4.43 \pm 1.44$ & \multirow{4}{*}{$1.409 \& 0.340$} \\
\hline & After & $1.41 \& 0.72$ & $1.81 \& .76$ & \\
\hline & Before-after & $2.20 \& 1.60$ & $1.36 \& 0.52$ & \\
\hline & $t \& p$ & $4.263 \& 0.003^{*}$ & $4.345 \& 0.004^{*}$ & \\
\hline \multirow{4}{*}{ NDI } & Before & $7.15 \pm 1.34$ & $7.20 \& 1.54$ & \multirow{4}{*}{$-0.6790 .351$} \\
\hline & After & $4.01 \pm 1.23$ & $2.45 \& 2.35$ & \\
\hline & Before-after & $3.72 \& 2.57$ & $2.30 \& 2.42$ & \\
\hline & $t \& p$ & $3.52 \pm 0.003^{*}$ & $2.760 \pm 0.042^{*}$ & \\
\hline \multirow{4}{*}{ Algometer Rt } & Before & $9.61 \& 1.26$ & $7.46 .5 \& 4.31$ & \multirow{4}{*}{$1.024 \& .0 .438$} \\
\hline & After & $13.01 \& 2.80$ & $14.1 \& 3.61$ & \\
\hline & Before-after & $1.89 \pm 0.29$ & $2.72 \pm .1 .47$ & \\
\hline & $t \& p$ & $3.478 \& .005^{*}$ & $3.4211 \& 0.006^{*}$ & \\
\hline \multirow{4}{*}{ Algometer Lt } & Before & $8.32 \pm 2.13$ & $7.93 \pm 2.82$ & \multirow{4}{*}{$0.31 \& 0.725$} \\
\hline & After & $13.64 \pm 3.15$ & $13.20 \pm 4.38$ & \\
\hline & Before-after & $2.62 \pm 0.62$ & $2.37 \& 2.36$ & \\
\hline & $t \& p$ & $3.127 \& 0.001^{*}$ & $2.412 \& 0.003$ & \\
\hline
\end{tabular}

Values are presented as mean (standard deviation). Lt: left, Rt: right, t-test ( $\mathrm{t}), \mathrm{P}$ (probability value) * indicates significant value $(P<0.05)$

\section{DISCUSSION}

Therapeutic exercises have been used to manage patients with MND. However, there is still a need to determine the most effective exercise approach for patients with MND. Therefore, this study was done to compare between the effects of stabilization and McKenzie exercises on pain perception, disability, pain threshold, and cervical ROM in patients with MND. According to the results of this study, both intervention protocols provided statistical significant improvements in each group. However, there were no statistically significant differences between both groups.

Patients in the first (stabilization) group experienced positive improvements in many parameters (pain intensity, disability, pain threshold, cervical extension and lateral neck flexion). The logical explanation is that pain inhibits the deep stabilizers, creating imbalance around the spine which precipitates the spine to further strain and pain [17]. Stabilization rectifies the longus capitis and the longus colli, which are imperative for cervical stability $[18,20]$. Some authors showed increased endurance of those muscles and reduced electromygoraphic activities of the scalene and sternocleidomastoid muscles in the stabilization group when contrasted with the conservative physical therapy program [21]. Those exercises may have induced local mechanical hypoalgesic responses [22]. Further, stabilization exercises have been shown to change the motor programing of the automatic feed-forward recruitment of the deep core spinal muscles [23].

Our results agreed with those of Dusunceli et al. (2009) who got significant pain relief and functional improvement in patients with chronic neck pain at 12 months after intervention with cervical stabilization exercises [24]. The present study findings also agreed with those of Cho (2011) who showed that cervical stabilization exercises reduced pain, increased maximum cervical muscle strength, endurance, ROM and muscular cross-sectional areas in chronic neck pain patients [25].

There has been concurrence of findings of this study with those of another research that indicated that neck stabilization exercises improved pain, disability and quality of life in patients with cervical radiculopathy [26]. Findings of this study also correlated with those of Celenay et al. (2016) who showed that stabilization exercises combined with manual therapy were superior to stabilization exercises alone for improving pain, disability, range of motion and quality of life in mechanical neck pain [27]. 
Participants of the second (McKenzie) group showed significant improvements in pain, disability, pain threshold and ROM of the neck except rotation. Improvements might be attributed to treatment of versatile shortening in the cervical spine that created constrained painful development and diminished spinal mobility which requires the utilization of Movements that support the procedure of remodeling. Just with the utilization of such loading strategies as McKenzie method, normal tissue function was re-set up [28]. The McKenzie method is a gentle approach directly performed by patients producing power utilizing repeated movement to end of range of motion in a direction that assuages the patient's side effects [29]. Patients also showed reduction of disability due to marked modifications in the cognitive and sensory perception of pain. McKenzie directional preference exercises may have improved selfconfidence, anxiety and fear of movements [29].

There are no similar studies investigating the efficacy of McKenzie exercises on MND while there are other studies sharing the same principal but pertaining to a related area (lumber spine). For instance, Garcia et al., (2013) showed concurrence with this study as they reported that McKenzie method was more effective than Back School method for promoting physical function in patients with low back dysfunction [10].

Regarding the neck region, there were studies that demonstrated the efficacy of McKenzie approach on cervical radiculopathy. For example, Abdulwahab et al. (2000) stated that doing repetitive neck retractions reduced radiculopathy pain in patients with cervical radiculopathy [30]. On accordance with these study results Murtezani et al. (2015) reported that McKenzie intervention indicates significant change in spinal movement, functional capacity and pain relief among patients with chronic LBP [31]. Likewise, McKenzie's method was more effective than lumbar strengthening exercises in treating non-specific low back pain patients [32].

The author of this study did not find differences between both programs. This was consistent with the findings of Miller et al. in a different area (the lumbar region) who failed to find differences between stabilization exercises and
McKenzie exercises in reducing pain and disability for patients with chronic low back pain [11]. This might be due to some limitations that this study pertains.

On the other hand, other authors reported improved pain, disability, and thickness of the transverse abdominis following stabilization exercises when compared with the McKenzie group in nonspecific chronic low back pain [33]. The opposite was found in another study where McKenzie's exercises were more effective than stabilization exercises in reducing pain and improving function in chronic low back pain [34]. Therefore, further comparative studies are warranted to measure which approach is more effective for the appropriate subgroups of patients.

Limitations of the study include the following: first, the sample size was small. Therefore, the results cannot be generalized. Second, the daily living activities of the participants were not completely controlled which is difficult to overcome. Third, there were no measurements of long-term and psychological outcomes. Fourth, patients may have not adhered to the home exercise program. Finally, yet importantly, the author did not utilize the electromyography to assess muscle recruitment during exercise performance. Further studies are still needed to determine the optimum dose for both protocols.

\section{CONCLUSION}

These study outcomes showed that stabilization and McKenzie exercise equally improved pain perception, disability, pain threshold and ROM of patients with MND.

\section{ACKNOWLEDGEMENTS}

The author would like to all the patients who kindly accepted voluntarily to participate in the study.

\section{Conflicts of interest: None}

\section{REFERENCES}

[1]. Lauche R, Cramer H, Choi KE, Rampp T, Saha FJ, Dobos GJ, et al. The influence of a series of five dry cupping treatments on pain and mechanical thresholds in patients with chronic non-specific neck pain - a randomized controlled pilot study. BMC Complement Altern Med 2011; 11:63. 
[2]. Hogg-Johnson S, van der Velde G, Carroll LJ, Holm LW, Cassidy JD, Guzman J, Côté P et al. 2000-2010 Task Force on Neck Pain and Its Associated Disorders The burden and determinants of neck pain in the general population: results of the Bone and Joint Decade 2000-2010 Task Force on Neck Pain and Its Associated Disorders. Spine 2008; 33:39-51.

[3]. Kim D, Cho M, Park Y et al. Effect of an exercise program for posture correction on musculoskeletal pain. J Phys Ther Sci 2015; 27:1791-1794.

[4]. Yoo WG. Effect of the neck retraction taping (NRT) on forward head posture and the upper trapezius muscle during computer work. J Phys Ther Sci 2013; 25:581-582.

[5]. McKenzie R, May S. The cervical and thoracic spine. Mechanical diagnosis and therapy. Waikanae: New Zealand: Spinal Publications, 2008:1

[6]. Ilter L, Dilek B, Batmaz I, Ulu MA, Sariyildiz MA, Nas $K$, et al. Efficacy of pulsed and continuous therapeutic ultrasound in myofascial pain syndrome: $A$ randomized controlled study. Am J Phys Med Rehab 2015; 94(7):547-554.

[7]. Kim DH, Yoon KB, Park S, Jin TE, An YJ, Schepis EA, et al. Comparison of NSAID patch given as monotherapy and NSAID patch in combination with transcutaneous electric nerve stimulation, a heating pad, or topical capsaicin in the treatment of patients with myofascial pain syndrome of the upper trapezius: a pilot study. Pain Med 2014; 15(12): 2128-2138.

[8]. Im B, Kim Y, Chung Y and Hwang S. Effects of scapular stabilization exercise on neck posture and muscle activation in individuals with neck pain and forward head posture. J Phys Ther Sci 2016; 28: 951955.

[9]. Gross AR, Paquin JP, Dupont G, et al. See comment in PubMed Commons belowExercises for mechanical neck disorders: A Cochrane review update. Man Ther 2016; 24:25-45.

[10]. Garcia AN, Costa LC, da Silva T, Gondo TF, Cyrillo, FN. Effectiveness of back school versus McKenzie exercises in patients with chronic nonspecific low back pain: A randomized control trial. Physical Therapy 2013; 93(6):729-747.

[11]. Miller ER, Schnek RJ, Karnes JL, Rousselle JG. A comparison of the McKenzie approach to a specific spine stabilization program for chronic low back pain. Journal of Manual and Manipulative Therapy 2005; 12(2):103-112.

[12].McKenzie Institute. 2013. http:// www.mckenziemdt.org/.Sept 22, 2013.

[13]. Mitchell U, Myrer JW, Johnson AW, Hilton SC. Restless leg syndrome and near-infrared light: An alternative treatment option. Physiother Theory Pract 2010; 15(3):1-7.

[14]. Saltychev M, Mattie R, McCormick Z, Laimi K. Psychometric properties of the a. neck disability index amongst patients with chronic neck pain using item response theory. Disabil Rehabil 2017; 13:1-6.

[15]. Fischer AA. Pressure threshold meter: its use for quantification of tender spots. Arch Phys Med
1986; 67:836-838.

[16]. Barlow Y, Willoughby J. Pathophysiology of soft tissue repair. Br Med Bul. 1992; 148.698-711.

[17]. Jull G, Falla D, Hodges P, et al.: Cervical flexor muscle retraining: physiological mechanisms of efficacy. Paper presented at: 2nd International Conference on Movement Dysfunction, 2005; 23-25.

[18]. Jull G, Kristjansson E, Dall'Alba P. Impairment in the cervical flexors: a comparison of whiplash and insidious onset neck pain patients. Man Ther 2004; 9: 89-94.

[19]. Clare HA, Adams R, Maher CG. Reliability of McKenzie classification of patients with cervical or lumbar pain. J Manipulative Physiol Ther 2005; 28:122-127.

[20]. Lee S, Park J, Lee D. The effects of cervical stabilization exercises on the electromyographic activity of shoulder stabilizers. J Phys Ther Sci 2013; 25: 15571560.

[21]. Ghaderi F, Jafarabadi MA, Javanshir K. The clinical and EMG assessment of the effects of stabilization exercise on nonspecific chronic neck pain: A randomized controlled trial. J Back Musculoskelet Rehabil 2017; 30(2):211-219.

[22]. O'Leary S, Falla D, Hodges PW, et al. Specific therapeutic exercise of the neck induces immediate local hypoalgesia. J Pain 2007; 8:832-839.

[23]. Millisdotter M, Strömqvist B. Early neuromuscular customized training after surgery for lumbar disc herniation: a prospective controlled study. Eur Spine J 2007; 16:19-26.

[24]. Dusunceli Y, Ozturk C, Atamaz F, et al. Efficacy of neck stabilization exercises for neck pain: a randomized controlled study. J Rehabil Med, 2009; 41: 626-631.

[25]. Cho HY: Effects of cervical stabilization exercise type on muscle strength and endurance, cross sectional area of cervical in patients with chronic cervical pain. Korea University, Dissertation of doctor's degree, 2011.

[26].Gelecek N, Akkan H. The effect of stabilization exercise training on pain and functional status in patients with cervical radiculopathy. J Back Musculoskelet Rehabil. 2017; 8. doi: 10.3233/BMR169583.

[27]. Celenay ST, Akbayrak T, and Kaya DO. A comparison of the effects of stabilization exercises plus manual therapy to those of stabilization exercises alone in patients with nonspecific mechanical neck pain: a randomized clinical trial. J Ortho Sports Phys Ther 2016; 46(2):44-55.

[28]. McKenzie R, May S. The Human Extremities: Mechanical Diagnosis \& Therapy. Spinal Publications, New Zealand. Volume two PO Box 2026, Raumati Beach, New Zealand. 2000; pp.255-321.

[29]. Petersen T, Kryger P, Ekdahl C, Olsen S and Jacobsen S. The effect of McKenzie therapy as compared with that of intensive strengthening training for the treatment of patients with sub-acute or chronic low back pain: a randomized controlled trial. Spine 2002; (27):1702-1709. 
[30]. Abdulwahab PS, Sabbahi M. Neck retraction, cervical root decompression, and radicular pain. Journal of Orthopedic Sports Physical Therapy 2000; 30(1):4-12.

[31]. Murtezani A, Govori V, Meka VS, Ibraimi Z, Rrecaj S and Gashi S. Comparison of McKenzie therapy with electrophysical agents for the treatment of work related low back pain: A randomized controlled trial. J Back Musculoskeletal Rehabil. 2015;28(2):247253.

[32]. Browder DA, Childs JD, Cleland JA, et al. Effectiveness of an extension-oriented treatment approach in a subgroup of subjects with low back pain: a randomized clinical trial. Phys Ther 2007; 87:16081618.
[33]. Hosseinifar M, Akbari M, Behtash H, Amiri M. The effects of stabilization and Mckenzie exercises on transverse abdominis and multifidus muscle thickness, pain, and disability: A randomized controlled trial in nonspecific chronic low back pain. J Phys Ther Sci 2013; 25:1541-1545.

[34]. Alessandra NG, Francine LB, Gondo RA, Renata AC, Leonardo OP. Effects of two physical therapy interventions in patients with chronic non-specific low back pain, feasibility of a randomized controlled trial. Rev Bras Fisioter, São Carlos; Sept./Oct 2011;15(5):420-427.

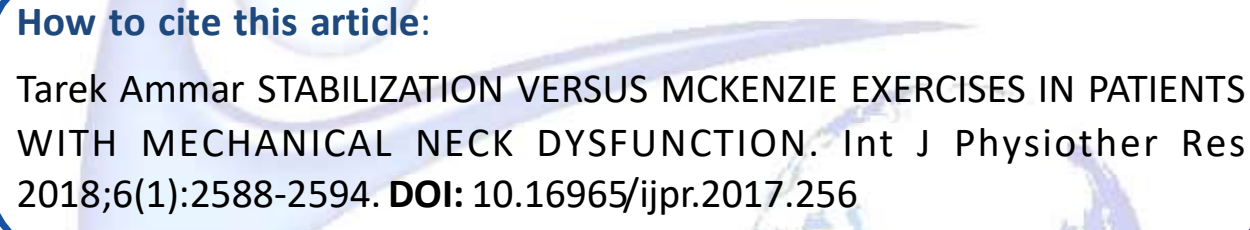

